\title{
Reiterated homogenization applied in hydrodynamic lubrication
}

\author{
A Almqvist $^{1}$, E K Essel $^{2,3}$, Fabricius $^{2 *}$ and $\mathbf{P}$ Wall $^{2}$ \\ ${ }^{1}$ Division of Machine Elements, Luleå University of Technology, Luleå, Sweden \\ ${ }^{2}$ Department of Mathematics, Luleå University of Technology, Luleå, Sweden \\ ${ }^{3}$ Department of Mathematics and Statistics, University of Cape Coast, Cape Coast, Ghana
}

The manuscript was received on 11 March 2008 and was accepted after revision for publication on 9 June 2008.

DOI: $10.1243 / 13506501 J E T 426$

\begin{abstract}
This work is devoted to studying the combined effect that arises due to surface texture and surface roughness in hydrodynamic lubrication. An effective approach in tackling this problem is by using the theory of reiterated homogenization with three scales. In the numerical analysis of such problems, a very fine mesh is needed, suggesting some type of averaging. To this end, a general class of problems is studied that, e.g. includes the incompressible Reynolds problem in both artesian and cylindrical coordinate forms. To demonstrate the effectiveness of the method several numerical results are presented that clearly show the convergence of the deterministic solutions towards the homogenized solution. Moreover, the convergence of the friction force and the load carrying capacity of the lubricant film is also addressed in this paper. In conclusion, reiterated homogenization is a feasible mathematical tool that facilitates the analysis of this type of problem.
\end{abstract}

Keywords: Reynolds equation, reiterated homogenization, surface roughness and texture

\section{INTRODUCTION}

Throughout the years, the general theory of homogenization has been successfully applied to different problems connected to hydrodynamic lubrication, see e.g. [1-7]. In these works it was shown that the rapid oscillations (in the coefficients of the Reynolds type equation under consideration) induced by the surface roughness, could efficiently be averaged by the homogenization method employed. In these previous results, it is assumed that the lubrication problem exhibits two separable scales, i.e. a global scale describing the geometric shape of the application and a local scale describing the surface roughness.

In the present work, it is assumed that the problem of interest, exhibits three separable scales, i.e. one global scale describing geometry, one oscillating local scale describing the surface texture, and a faster oscillating local scale describing the surface roughness.

\footnotetext{
*Corresponding author: Department of Mathematics, Luleå University of Technology, Luleå, SE-971 87, Sweden. email: John.Fabricius@ltu.se
}

Homogenization of problems with two or more oscillating scales are referred to as reiterated homogenization, see e.g. [8-10]. In this paper, a generalized form of the Reynolds problem is considered, governing incompressible and Newtonian flow, with the advantage to unify both the Cartesian and the cylindrical coordinate formulations. In particular, the aim is to obtain a general homogenized problem that corresponds to a class of problems modelled by equation (1). One technique within the homogenization theory is the formal method of multiple scale expansion, see e.g. $[\mathbf{8}, 11]$. To accomplish this aim the formal method of multiple scale expansion is employed to obtain a homogenized problem (8) for equation (1). For other problems connected to the incompressible Reynolds that have been studied by multiple scale expansion see [1, 3, 7].

By means of numerical analysis, the convergence, of the direct numerical solution towards the homogenized counterpart, in terms of load carrying capacity and hydrodynamically induced friction is quantified. The results show that the combined effect due to the texture and roughness on a modelled bearing can be effectively analysed through reiterated homogenization. More specifically, the discrepancies, between the 
proposed method and the direct numerical approach, in terms of predicted load carrying capacity and friction force are tolerably small; $\mathcal{O}$ ( 1 per cent) for textures as well as roughness of wavelengths likely to be found in a real application. That is, wavelengths within the ranges $1 / 100-1 / 10$ of the length bearing for the texture and $1 / 10000-1 / 100$ for the roughness.

\section{THE HOMOGENIZATION PROCEDURE}

In this section, a class of equations that includes the Reynolds equation governing incompressible Newtonian flow is considered. It can be seen that the generalized form (1), makes it possible to study the Reynolds problem in its Cartesian, and cylindrical coordinate forms. (See section 4 ).

Let $\Omega$ be an open bounded subset of $\mathbb{R}^{2}, Y=(0,1)^{2}$, and $Z=(0,1)^{2}$. Introduce the auxiliary matrix $\mathbf{A}=$ $\left(a_{i j}\right)$, where $a_{i j}=a_{i j}(x, y, z)$, and $i=1,2$, and $j=1,2$ are smooth functions that are $Y$-periodic in $y$ and $Z$-periodic in $z$. It is also assumed that a constant $\alpha>0$ exists such that

$$
\sum_{i, j=1}^{2} a_{i j}(x, y, z) \xi_{i} \xi_{j} \geqslant \alpha|\xi|^{2} \text { for every } \xi \in \mathbb{R}^{2}
$$

Moreover, the auxiliary vector $\boldsymbol{b}=\left(b_{i}\right)$ is introduced, where $b_{i}=b_{i}(x, y, z)$ and $i=1,2$, are smooth functions that are $Y$-periodic in $y$ and $Z$-periodic in $z$. Let $\varepsilon>0$ and define the matrix $\mathbf{A}_{\varepsilon}$ and the vector $\boldsymbol{b}_{\varepsilon}$ as

$$
\begin{aligned}
\mathbf{A}_{\varepsilon}(x) & =\left(\begin{array}{ll}
a_{11}^{\varepsilon}(x) & a_{12}^{\varepsilon}(x) \\
a_{21}^{\varepsilon}(x) & a_{22}^{\varepsilon}(x)
\end{array}\right)=\mathbf{A}\left(\frac{x, x}{\varepsilon},\left(\frac{x}{\varepsilon}\right)^{2}\right) \\
\boldsymbol{b}_{\varepsilon}(x) & =\left(\begin{array}{l}
b_{1}^{\varepsilon}(x) \\
b_{2}^{\varepsilon}(x)
\end{array}\right)=\boldsymbol{b}\left(x, \frac{x}{\varepsilon},\left(\frac{x}{\varepsilon}\right)^{2}\right)
\end{aligned}
$$

Consider the following boundary value problem

$$
\begin{aligned}
\nabla_{x} \cdot\left(\mathbf{A}_{\varepsilon}(x) \nabla_{x} p_{\varepsilon}(x)\right) & =\nabla_{x} \cdot \boldsymbol{b}_{\varepsilon}(x) \text { in } \Omega \\
p_{\varepsilon}(x) & =0 \text { on } \partial \Omega
\end{aligned}
$$

For small values of the parameter $\varepsilon$, the coefficients in equation (1) are rapidly oscillating. This suggests some type of asymptotic analysis; $p_{\varepsilon} \rightarrow p_{0}$ as $\varepsilon \rightarrow 0$ and $p_{0}$ can be found by solving a so-called homogenized equation (8), which does not contain any rapid oscillations. This means that $p_{0}$ may be used as an approximation of the solution $p_{\varepsilon}$ for small values of $\varepsilon$.

The method of multiple scale expansion developed in the homogenization theory is used to derive a homogenization result connected to equation (1). For general information concerning this method in connection to homogenization, see e.g. $[\mathbf{8}, 11]$. The homogenization of Reynolds type equations involving only one local scale have been studied by multiple scale expansion in references [1], [3], [7], and [12].
Assume that $p_{\varepsilon}$ is of the form

$$
p_{\varepsilon}(x)=\sum_{i=0}^{\infty} \varepsilon^{i} p_{i}\left[x, \frac{x}{\varepsilon}, \frac{x^{2}}{\varepsilon}\right]
$$

where $p_{i}=p_{i}(x, y, z)$ is $Y$-periodic in $y$ and $Z$-periodic in $z$. The main idea is to insert the expansion (2) into equation (1), and then collect terms of the same order of $\varepsilon$ and analyse the system of equations obtained. A comprehensive analysis can be found in Appendix 2 . The main result is that the leading term $p_{0}$ in the expansion (2) is of the form $p_{0}=p_{0}(x)$ and is found by the following homogenization algorithm:

Step 1: solve the local problems (on the $z$-scale)

$$
\begin{aligned}
& \nabla_{z} \cdot\left(\mathbf{A}\left(\nabla_{z} u_{i}+e_{i}\right)\right)=0 \text { in } Z, \quad(i=1,2) \\
& \nabla_{z} \cdot\left(\mathbf{A} \nabla_{z} u_{0}-\boldsymbol{b}\right)=0 \text { in } Z
\end{aligned}
$$

where $u_{i}=u_{i}(x, y, z), i=0,1,2$, is $Y$-periodic in $y$, $Z$-periodic in $z$, and $\left\{e_{1}, e_{2}\right\}$ is the canonical basis in $\mathbb{R}^{2}$. Use these local solutions to define the matrix

$$
\mathcal{A}=\mathcal{A}(x, y, z)=\left(\begin{array}{cc}
1+\frac{\partial u_{1}}{\partial z_{1}} & \frac{\partial u_{2}}{\partial z_{1}} \\
\frac{\partial u_{1}}{\partial z_{2}} & 1+\frac{\partial u_{2}}{\partial z_{2}}
\end{array}\right)
$$

Step 2: solve the local problems (on the $y$-scale)

$$
\begin{aligned}
& \nabla_{y} \cdot\left[\overline{\mathbf{A} \mathcal{A}}^{z}\left(\nabla_{y} v_{i}+e_{i}\right)\right]=0 \text { in } Y,(i=1,2) \\
& \nabla_{y} \cdot\left[\overline{\mathbf{A} \mathcal{A}}^{z} \nabla_{y} v_{0}-\left({\overline{\boldsymbol{b}-\mathbf{A} \nabla_{z} u_{0}}}^{z}\right)\right]=0 \text { in } Y
\end{aligned}
$$

where $v_{i}=v_{i}(x, y), i=0,1,2$, is $Y$-periodic in $y$ and $\overline{\mathbf{A A}}^{z}$ is the average with respect to $Z$. Use these local solutions to define the matrix

$$
\mathcal{B}=\mathcal{B}(x, y)=\left(\begin{array}{cc}
1+\frac{\partial \nu_{1}}{\partial y_{1}} & \frac{\partial \nu_{2}}{\partial y_{1}} \\
\frac{\partial \nu_{1}}{\partial y_{2}} & 1+\frac{\partial \nu_{2}}{\partial y_{2}}
\end{array}\right)
$$

Step 3: compute the homogenized matrix $\mathbf{A}_{0}$ and the homogenized vector $\boldsymbol{b}_{0}$ by the following formulas

$$
\mathbf{A}_{0}(x)={\overline{\overline{\mathbf{A} A \mathcal{B}}^{z}}}^{y} \text { and } \boldsymbol{b}_{0}(x)={\overline{\boldsymbol{b}-\mathbf{A} \nabla_{z} u_{0}-\mathbf{A} \mathcal{A} \nabla_{y} v_{0}}}^{y}
$$

Step 4: find $p_{0}$ by solving the so called homogenized problem

$$
\begin{aligned}
& \nabla_{x} \cdot\left(\mathbf{A}_{0}(x) \nabla_{x} p_{0}(x)\right)=\nabla_{x} \cdot \boldsymbol{b}_{0}(x) \text { in } \Omega \\
& p_{0}(x)=0 \quad \text { on } \partial \Omega
\end{aligned}
$$


The main advantage of the above algorithm is that the scales are treated separately, i.e. first one 'averages' with respect to the $z$-scale, then with respect to the $y$-scale and finally one solves the homogenized equation. It is noted that the homogenized equation does not contain any oscillating coefficents, nevertheless, it takes into account the effects of the local scales, see equation (7). The fact that the scales can be separated in this way, significantly simplifies the numerical analysis of the problem.

\section{AN ADDITIONAL RESULT}

In this section, the convergence of $\nabla p_{\varepsilon}$ is investigated. The functions $p_{i}, i=0,1,2$, in the expansion is of the form

$$
p_{0}=p_{0}(x), \quad p_{1}=p_{1}(x, y), \quad p_{2}=p_{2}(x, y, z)
$$

see Appendix 2. When inserted into equation (2)

$$
\nabla_{x} p_{\varepsilon}(x)=\nabla_{x} p_{0}(x)+\nabla_{y} p_{1}(x, y)+\nabla_{z} p_{2}(x, y, z)+\varepsilon[\ldots]
$$

which means that

$$
\nabla_{x} p_{\varepsilon}(x) \approx \nabla_{x} p_{0}(x)+\nabla_{y} p_{1}(x, y)+\nabla_{z} p_{2}(x, y, z)
$$

for small values of $\varepsilon$. According to the analysis in Appendix 2, $p_{1}$ and $p_{2}$ can be expressed in terms of the solutions $u_{i}$ and $v_{i}$ of the local problems (3), (4), (5), and (6), respectively. Making use of equations (49) and (53) in addition to equations (45) and (58) yields

$$
\begin{aligned}
\nabla_{x} p_{\varepsilon}(x) \approx & \nabla_{z} u_{0}(x, y, z)+\mathcal{A}(x, y, z) \nabla_{y} v_{0}(x, y) \\
& +\mathcal{A}(x, y, z) \mathcal{B}(x, y) \nabla_{x} p_{0}(x)
\end{aligned}
$$

after some straightforward calculations. According to references $[\mathbf{1 0}],[\mathbf{1 3}]$, and $[\mathbf{1 4}]$, the following convergence holds

$$
\begin{aligned}
& \int_{\Omega} \nabla_{x} p_{\varepsilon}(x) \varphi\left(x, \frac{x}{\varepsilon},\left(\frac{x}{\varepsilon}\right)^{2}\right) \mathrm{d} x \longrightarrow \\
& \int_{\Omega} \int_{Y} \int_{Z}\left[\nabla_{z} u_{0}+\mathcal{A} \nabla_{y} \nu_{0}+\mathcal{A B} \nabla_{x} p_{0}\right] \\
& \quad \times \varphi(x, y, z) \mathrm{d} z \mathrm{~d} y \mathrm{~d} x
\end{aligned}
$$

for any smooth function $\varphi$ that is $Y$-periodic in $y$ and $Z$-periodic in $z$.

\section{APPLICATION TO HYDRODYNAMIC LUBRICATION}

In this section, it is studied how the general reiterated homogenization result can be applied to analyse the effects of texture and surface roughness in the hydrodynamic lubrication goverened by the Reynolds equation. For this purpose, an auxiliary function is introduced, which may be used to represent the lubricant film thickness

$$
h(x, y, z)=h_{0}(x)+h_{\mathrm{T}}(x, y)+h_{\mathrm{R}}(x, y, z),
$$

where

(a) $h_{0}(x)$ describes the geometry of the bearing;

(b) $h_{\mathrm{T}}(x, y)$ is a $Y$-periodic function in $y$, representing surface texture;

(c) $h_{\mathrm{R}}(x, y, z)$ is a $Y$-periodic function in $y$ and a $Z$-periodic in $z$, representing the roughness contribution.

Note that this formulation admits studying problem where the texture and the roughness changes with position at the tribological interface. For example, this enables studying the effects of a texture only on a part of the surface, which in turn may exhibit different surface roughness patterns at different parts of the texture itself. However, here the numerical examples are restricted to consider the case where the texture and the roughness representation does not change with the position, i.e. $h_{\mathrm{T}}=h_{\mathrm{T}}(y)$ and $h_{\mathrm{R}}=h_{\mathrm{R}}(z)$. By making use of the auxiliary function $h$, it is possible to model the deterministic film thickness $h_{\varepsilon}$ as

$$
h_{\varepsilon}(x)=h\left[x, \frac{x}{\varepsilon},\left(\frac{x}{\varepsilon}\right)^{2}\right]=h_{0}(x)+h_{\mathrm{T}}\left(\frac{x}{\varepsilon}\right)+h_{\mathrm{R}}\left(\frac{x}{\varepsilon}\right)^{2}
$$

where $\varepsilon$ is a parameter that describes the texture and roughness wavelength.

Now, by choosing

$$
\begin{aligned}
& \mathbf{A}_{\varepsilon}(x)=\left(\begin{array}{cc}
h_{\varepsilon}^{3}(x) & 0 \\
0 & h_{\varepsilon}^{3}(x)
\end{array}\right) \\
& \boldsymbol{b}_{\varepsilon}(x)=6 \mu U h_{\varepsilon}(x) e_{1}
\end{aligned}
$$

in equation (1), where $e_{1}=(1,0)$, the Reynolds equation describing incompressible Newtonian flow in Cartesian coordinates is obtained, that is

$$
\begin{gathered}
\nabla_{x} \cdot\left[\left(\begin{array}{cc}
h_{\varepsilon}^{3} & 0 \\
0 & h_{\varepsilon}^{3}
\end{array}\right) \nabla_{x} p_{\varepsilon}\right]=6 \mu U \nabla_{x} \cdot\left(h_{\varepsilon} e_{1}\right) \quad \text { in } \Omega \\
p_{\varepsilon}(x)=0 \text { on } \partial \Omega
\end{gathered}
$$

where, $p_{\varepsilon}$ is the hydrodynamically induced pressure distribution, $\mu$ the (constant) viscosity of the Newtonian lubricant, and $U$ the linear speed of the moving smooth surface. 
It is also observed that by choosing

$$
\begin{aligned}
\mathbf{A}_{\varepsilon}(x) & =\left(\begin{array}{cc}
h_{\varepsilon}^{3}(x) / x_{2} & 0 \\
0 & x_{2} h_{\varepsilon}^{3}(x)
\end{array}\right) \\
\boldsymbol{b}_{\varepsilon}(x) & =6 \mu \omega x_{2} h_{\varepsilon}(x) e_{1}
\end{aligned}
$$

in equation (1), where $\omega$ is the angular speed of the smooth rotating surface and $\left(x_{1}, x_{2}\right)$ are the angular and the radial coordinates, the Reynolds equation describing incompressible Newtonian flow in cylindrical coordinates is obtained

$$
\begin{aligned}
& \nabla_{x} \cdot\left[\left(\begin{array}{cc}
h_{\varepsilon}^{3}(x) / x_{2} & 0 \\
0 & x_{2} h_{\varepsilon}^{3}(x)
\end{array}\right) \nabla_{x} p_{\varepsilon}\right] \\
& \quad=6 \mu \omega x_{2} \nabla_{x} \cdot\left(h_{\varepsilon} e_{1}\right) \quad \text { in } \Omega p_{\varepsilon}(x)=0 \text { on } \partial \Omega
\end{aligned}
$$

It should be noted that the homogenization result, which is that $p_{\varepsilon} \rightarrow p_{0}$ as $\varepsilon \rightarrow 0$, does not require any restrictions on the geometry, neither on the texture ( $y$-scale) nor on the roughness ( $z$-scale). The only limitation is that $\varepsilon$ should be sufficiently small in order to approximate the hydrodynamic pressure $p_{\varepsilon}$ with $p_{0}$. As will be seen this is actually no limitation since $\varepsilon$ is very small in realistic examples.

From the homogenization result, convergence of load carrying capacity $\mathcal{I}_{\varepsilon}$ automatically follows, that is

$$
\mathcal{I}_{\varepsilon}=\int_{\Omega} p_{\varepsilon}(x) \mathrm{d} x \longrightarrow \int_{\Omega} p_{0}(x) \mathrm{d} x=\mathcal{I}_{0}
$$

Moreover, the convergence of $\nabla p_{\varepsilon}$ in section 3 is studied. The convergence of hydrodynamically induced friction force, $\mathcal{F}_{\varepsilon}$, and frictional torque, $\mathcal{T}_{\varepsilon}$, are connected to the derivative $\partial p_{\varepsilon} / \partial x_{1}$, and by making use of equation (10), the following expressions are obtained

$$
\begin{aligned}
& \mathcal{F}_{\varepsilon}=\int_{\Omega}\left(\frac{\mu \mathrm{U}}{h_{\varepsilon}(x)}+\frac{h_{\varepsilon}(x)}{2} \frac{\partial p_{\varepsilon}}{\partial x_{1}}\right) \mathrm{d} x \longrightarrow \\
& \mathcal{F}_{0}=\int_{\Omega} \int_{Y} \int_{Z}\left(\frac{\mu \mathrm{U}}{h(x, y, z)}+\frac{h(x, y, z)}{2} \frac{\partial p_{0}}{\partial x_{1}}\right) \mathrm{d} z \mathrm{~d} y \mathrm{~d} x \\
& +\int_{\Omega} \int_{Y} \int_{Z} \frac{h(x, y, z)}{2}\left[\frac{\partial u_{0}}{\partial z_{1}}+\frac{\partial u_{1}}{\partial z_{1}} \frac{\partial p_{0}}{\partial x_{1}}\right. \\
& \left.+\frac{\partial u_{2}}{\partial z_{2}} \frac{\partial p_{0}}{\partial x_{2}}\right] \mathrm{d} z \mathrm{~d} y \mathrm{~d} x+\int_{\Omega} \int_{Y} \int_{Z} \frac{h(x, y, z)}{2} \\
& \times\left[\frac{\partial v_{0}}{\partial y_{1}}+\frac{\partial v_{1}}{\partial y_{1}} \frac{\partial p_{0}}{\partial x_{1}}+\frac{\partial v_{2}}{\partial y_{2}} \frac{\partial p_{0}}{\partial x_{2}}\right] \mathrm{d} z \mathrm{~d} y \mathrm{~d} x \\
& +\int_{\Omega} \int_{Y} \int_{Z} \frac{h(x, y, z)}{2}\left[( \begin{array} { l } 
{ \frac { \partial u _ { 1 } } { \partial z _ { 1 } } } \\
{ \frac { \partial u _ { 2 } } { \partial z _ { 1 } } }
\end{array} ) \cdot \left(\left(\begin{array}{l}
\frac{\partial v_{0}}{\partial y_{1}} \\
\frac{\partial v_{0}}{\partial y_{2}}
\end{array}\right)\right.\right. \\
& \left.\left.+\left(\begin{array}{ll}
\frac{\partial v_{1}}{\partial y_{1}} & \frac{\partial v_{2}}{\partial y_{1}} \\
\frac{\partial v_{1}}{\partial y_{2}} & \frac{\partial v_{2}}{\partial y_{2}}
\end{array}\right)\left(\begin{array}{l}
\frac{\partial p_{0}}{\partial x_{1}} \\
\frac{\partial p_{0}}{\partial x_{2}}
\end{array}\right)\right)\right] \mathrm{d} z \mathrm{~d} y \mathrm{~d} x
\end{aligned}
$$

for friction force and

$$
\begin{aligned}
& \mathcal{T}_{\varepsilon}=\int_{\Omega} x_{2}\left(\frac{\mu \omega x_{2}}{h_{\varepsilon}(x)}+\frac{h_{\varepsilon}(x)}{2 x_{2}} \frac{\partial p_{\varepsilon}}{\partial x_{1}}\right) x_{2} \mathrm{~d} x_{1} \mathrm{~d} x_{2} \longrightarrow \\
& \mathcal{T}_{0}=\int_{\Omega}\left\{\int_{Y} \int_{Z} x_{2}\left(\frac{\mu \omega x_{2}}{h(x, y, z)}+\frac{h(x, y, z)}{2 x_{2}} \frac{\partial p_{0}}{\partial x_{1}}\right) \mathrm{d} z \mathrm{~d} y\right\} \\
& \times x_{2} \mathrm{~d} x_{1} \mathrm{~d} x_{2}+\int_{\Omega}\left\{\int _ { Y } \int _ { Z } \frac { h ( x , y , z ) } { 2 } \left[\frac{\partial u_{0}}{\partial z_{1}}\right.\right. \\
& \left.\left.+\frac{\partial u_{1}}{\partial z_{1}} \frac{\partial p_{0}}{\partial x_{1}}+\frac{\partial u_{2}}{\partial z_{2}} \frac{\partial p_{0}}{\partial x_{2}}\right] \mathrm{~d} z \mathrm{~d} y\right\} x_{2} \mathrm{~d} x_{1} \mathrm{~d} x_{2} \\
& +\int_{\Omega}\left\{\int _ { Y } \int _ { Z } \frac { h ( x , y , z ) } { 2 } \left[\frac{\partial v_{0}}{\partial y_{1}}+\frac{\partial v_{1}}{\partial y_{1}} \frac{\partial p_{0}}{\partial x_{1}}\right.\right. \\
& \left.\left.+\frac{\partial v_{2}}{\partial y_{2}} \frac{\partial p_{0}}{\partial x_{2}}\right] \mathrm{~d} z \mathrm{~d} y\right\} x_{2} \mathrm{~d} x_{1} \mathrm{~d} x_{2} \\
& +\int_{\Omega}\left\{\int _ { Y } \int _ { Z } \frac { h ( x , y , z ) } { 2 } \left[( \begin{array} { l } 
{ \frac { \partial u _ { 1 } } { \partial z _ { 1 } } } \\
{ \frac { \partial u _ { 2 } } { \partial z _ { 1 } } }
\end{array} ) \cdot \left(\left(\begin{array}{l}
\frac{\partial v_{0}}{\partial y_{1}} \\
\frac{\partial v_{0}}{\partial y_{2}}
\end{array}\right)\right.\right.\right. \\
& \left.\left.+\left(\begin{array}{ll}
\frac{\partial v_{1}}{\partial y_{1}} & \frac{\partial v_{2}}{\partial y_{1}} \\
\frac{\partial v_{1}}{\partial y_{2}} & \frac{\partial v_{2}}{\partial y_{2}}
\end{array}\right)\left(\begin{array}{l}
\frac{\partial p_{0}}{\partial x_{1}} \\
\frac{\partial p_{0}}{\partial x_{2}}
\end{array}\right)\right) \mathrm{d} z \mathrm{~d} y\right\} x_{2} \mathrm{~d} x_{1} \mathrm{~d} x_{2}
\end{aligned}
$$

for frictional torque. To clarify, from the equations above, the resulting homogenized quantity is made up of friction force/torque due to the smooth (averaged) film thickness plus a corrector term identified by three separate contributions, i.e. due to roughness or texture acting alone or roughness and texture acting together.

In the following, numerical investigations are conducted to the convergence associated with load carrying capacity and the hydrodynamically induced friction force by employing a second-order finitedifference scheme. The results of these investigations, justify the applicability of the homogenization process presented in this paper. Subsequently, the effects of periodic texture and surface roughness are studied by considering a thrust pad bearing problem. It is observed that for one-dimensional texture and roughness representation only very small differences exist between the homogenized numerical solution (HNS) and the direct numerical solution (DNS). It is pointed out that it is only possible to find the DNS in the case of transversal and longitudinal (i.e. one-dimensional) texture and roughness due to the enormous number of discretization points that is required in the general case. From the general analysis, it is clear that it is always possible to obtain an approximate solution $p_{0}$ of $p_{\varepsilon}$, with very high accuracy by solving the homogenized equation. From an application point of view, this means that for arbitrary (i.e. also two-dimensional) yet physically relevant, texture and roughness, a highly accurate approximation $p_{0}$ of the pressure solution $p_{\varepsilon}$, 
can be obtained by solving the homogenized equation. This is one of the benefits with the method.

\subsection{A numerical investigation of convergence}

Computationally, it is extremely demanding to retrieve the DNS for short wavelength roughness (and texture). Therefore, to assess and quantify the convergence, the one-dimensional problem was first revisited. This elementary problem constitutes an excellent benchmark for the implemented numerics, since it is possible to obtain closed form expressions for the coefficients in the homogenized equation. Specifically, a onedimensional representation of the Reynolds equation is obtained, for incompressible and Newtonian flow, in Cartesian coordinates by considering equation (14), that is

$$
\begin{aligned}
& \frac{\mathrm{d}}{\mathrm{d} x}\left(h_{\varepsilon}^{3}(x) \frac{\mathrm{d} p_{\varepsilon}}{\mathrm{d} x}(x)\right)=6 \mu U \frac{\mathrm{d} h_{\varepsilon}}{\mathrm{d} x}(x) \text { in } 0 \leqslant x \leqslant L \\
& p_{\varepsilon}(0)=p_{\varepsilon}(L)=0
\end{aligned}
$$

where $L$ is the length of the stationary surface exhibiting texture and roughness.

Through equation (11), the film thickness function of the modelled linear slider bearing, is described with

$$
\begin{aligned}
& h_{0}(x)=h_{\mathrm{min}}+\frac{h_{\mathrm{min}}}{4}\left(1-\frac{x}{L}\right) \\
& h_{\mathrm{T}}(s)=2 h_{\mathrm{R}}(s)=\frac{h_{\mathrm{min}}}{4}\left[\frac{1}{2}(1-\cos (2 \pi s))\right]
\end{aligned}
$$

where $h_{\text {min }}$ denotes the fixed minimum film thickness of the corresponding smooth problem, i.e. the problem with a smooth stationary surface as well as a smooth moving surface. To generalize the results, the dimensionless variables $X=x / L, H=h / h_{\min }$, and $P_{\varepsilon}=p_{\varepsilon} /\left(6 \mu U L / h_{\min }^{2}\right)$ were introduced to obtain a dimensionless Reynolds problem

$$
\begin{aligned}
& \frac{\mathrm{d}}{\mathrm{d} X}\left(H_{\varepsilon}^{3}(X) \frac{\mathrm{d} P_{\varepsilon}}{\mathrm{d} X}(X)\right)=\frac{\mathrm{d} H_{\varepsilon}}{\mathrm{d} X}(X), \text { in } 0 \leqslant X \leqslant 1 \\
& P_{\varepsilon}(0)=P_{\varepsilon}(1)=0
\end{aligned}
$$

The dimensionless representation of the auxiliary film thickness function is also presented, in terms of these dimensionless variables, that is

$$
\begin{aligned}
H(X, y, z)= & +\frac{1}{4}(1-X)+\frac{1}{4}\left[\frac{1}{2}(1-\cos (2 \pi y))\right] \\
& +\frac{1}{8}\left[\frac{1}{2}(1-\cos (2 \pi z))\right]
\end{aligned}
$$

The homogenized problem corresponding to equation (20) reads as

$$
\begin{aligned}
& \frac{\mathrm{d}}{\mathrm{d} X}\left(\frac{1}{\overline{\overline{H^{-3}(X, y, z)^{z^{y}}}} \frac{\mathrm{d} P_{0}}{\mathrm{~d} X}}\right) \\
& \quad=\frac{\mathrm{d}}{\mathrm{d} X}\left(\frac{\overline{\overline{H^{-2}(X, y, z)^{z^{y}}}}}{\overline{\overline{H^{-3}(X, y, z)^{z^{y}}}}}\right), \quad \text { in } 0 \leqslant X \leqslant 1 \\
& P_{0}(0)=P_{0}(1)=0
\end{aligned}
$$

Figure 1 illustrates the convergence of load carrying capacity $\mathcal{I}_{\varepsilon}$ towards $\mathcal{I}_{0}$ with decreasing $\varepsilon$. In fact, it is the measure

$$
\frac{\left|\mathcal{I}_{\varepsilon}-\mathcal{I}_{0}\right|}{\mathcal{I}_{0}}
$$

(equivalent to

$$
\frac{\int_{0}^{1}\left|P_{\varepsilon}(X)-P_{0}(X)\right| \mathrm{d} X}{\int_{0}^{1} P_{0}(X) \mathrm{d} X}
$$

if $P_{\varepsilon}, P_{0} \geqslant 0$ ) that is considered as being a function of $\varepsilon$ in the figure. When computing the DNS, $2^{5}$ discrete nodes were used to represent a single wavelength of the texture, e.g. for $\varepsilon=2^{-7}$, a total number of $\left(1 / 2^{-7}\right)^{2} 2^{5}=2^{19}$ grid nodes were used. As deduced from the figure, the rate of convergence is very close to linear, with the goodness of fit equaling 0.99 . To further elaborate on the convergence of $P_{\varepsilon}$ towards $P_{0}$, a set of DNS $\left(P_{\varepsilon}\right)$ and the HNS $\left(P_{0}\right)$ is illustrated in Fig. 2.

To facilitate the derivation of the specific version of equation (17) that corresponds to the onedimensional dimensionless form of equation (1), $\mathbf{A}_{\varepsilon}=$ $H_{\varepsilon}^{3}\left(x_{1}\right)$ and $\boldsymbol{b}_{\varepsilon}=H_{\varepsilon}\left(x_{1}\right)$ are first chosen. Then, owing

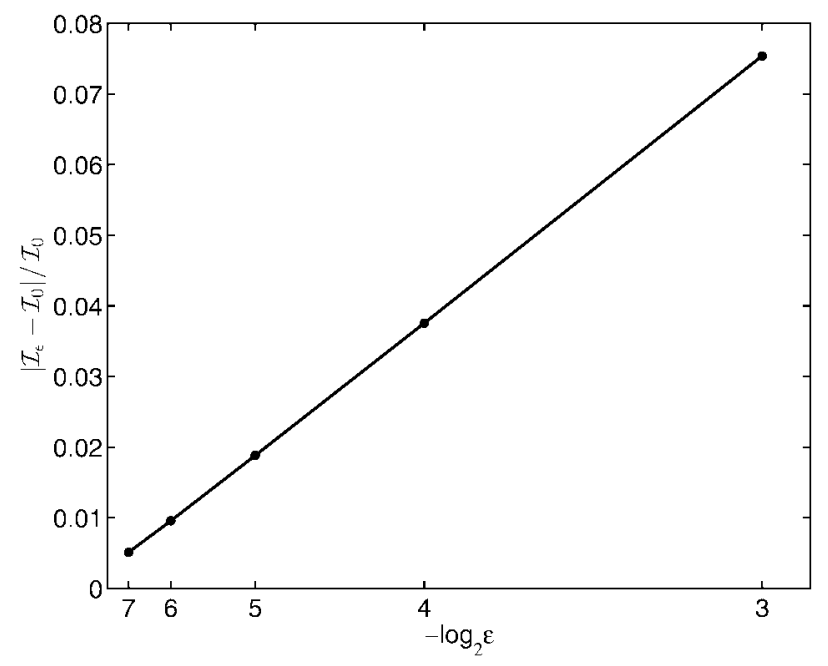

Fig. 1 Convergence of load carrying capacity $\mathcal{I}_{\varepsilon}$ towards $\mathcal{I}_{0}$ with decreasing $\varepsilon$ 


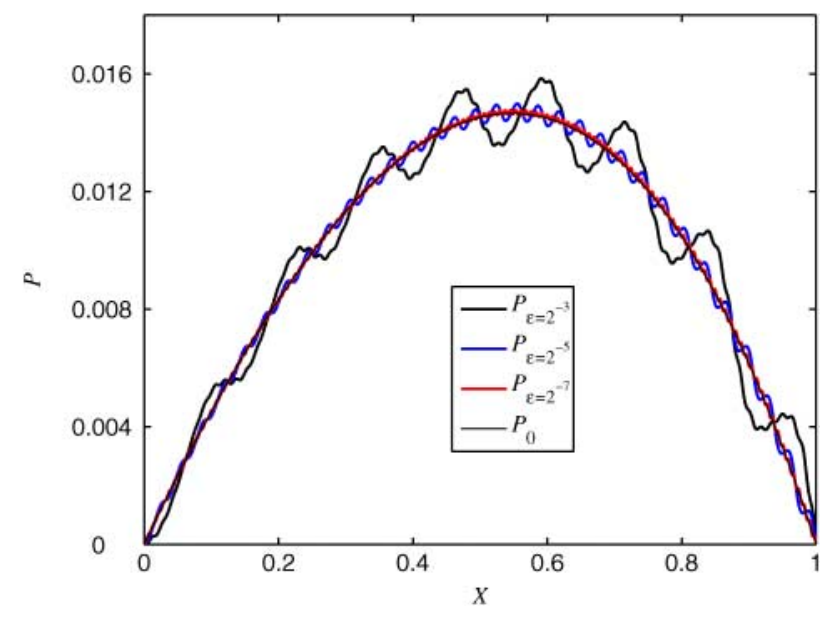

Fig. 2 A set of DNS $\left(P_{\varepsilon}\right)$ and the HNS $\left(P_{0}\right)$

to equation (17), the following convergence, in terms of dimensionless friction force $F_{\varepsilon}=\mathcal{F}_{\varepsilon} /\left(\mu U L / h_{\text {min }}\right)$ is obtained

$$
\begin{aligned}
F_{\varepsilon}= & \int_{0}^{1} \frac{1}{H_{\varepsilon}(X)}+3 H_{\varepsilon}(X) \frac{\mathrm{d} P_{\varepsilon}}{\mathrm{d} X}(X) \mathrm{d} X \rightarrow \\
F_{0}= & \int_{0}^{1} \int_{0}^{1} \int_{0}^{1}\left(\frac{1}{H}+3 H \frac{\mathrm{d} p_{0}}{\mathrm{~d} X}\right) \mathrm{d} z \mathrm{~d} y \mathrm{~d} X \\
& +\int_{0}^{1} \int_{0}^{1} \int_{0}^{1} 3 H\left(\frac{\partial u_{0}}{\partial z}+\frac{\partial u_{1}}{\partial z} \frac{\mathrm{d} p_{0}}{\mathrm{~d} X}\right) \mathrm{d} z \mathrm{~d} y \mathrm{~d} X \\
& +\int_{0}^{1} \int_{0}^{1} \int_{0}^{1} 3 H\left(\frac{\partial v_{0}}{\partial y}+\frac{\partial \nu_{1}}{\partial y} \frac{\mathrm{d} p_{0}}{\mathrm{~d} X}\right) \mathrm{d} z \mathrm{~d} y \mathrm{~d} X \\
& +\int_{0}^{1} \int_{0}^{1} \int_{0}^{1} 3 H\left[\frac{\partial u_{1}}{\partial z}\left(\frac{\partial v_{0}}{\partial y}+\frac{\partial \nu_{1}}{\partial y} \frac{\mathrm{d} p_{0}}{\mathrm{~d} X}\right)\right] \mathrm{d} z \mathrm{~d} y \mathrm{~d} X
\end{aligned}
$$

For the one-dimensional problem, the cell problems (3), (4), (5), and (6) can be solved explicitly. Inserting the solutions

$$
\begin{aligned}
\frac{\partial u_{0}}{\partial z} & =H^{-2}(X, y, z)-\frac{\overline{H^{-2}(X, y, z)^{z}}}{\overline{H^{-3}(X, y, z)^{z}}} H^{-3}(X, y, z) \\
\frac{\partial v_{0}}{\partial y} & =\overline{H^{-2}(X, y, z)^{z}}-\frac{\overline{\overline{H^{-2}(X, y, z)^{z^{y}}}}}{\overline{\overline{H^{-3}(X, y, z)^{z^{y}}}}} \overline{H^{-3}(X, y, z)^{z}} \\
\frac{\partial u_{1}}{\partial z} & =-1+\frac{\frac{H^{-3}(X, y, z)}{\overline{H^{-3}(X, y, z)^{z}}}}{\frac{\partial v_{1}}{\partial y}}=-1+\frac{\overline{H^{-3}(X, y, z)^{z}}}{\overline{\overline{H^{-3}(X, y, z)^{z^{y}}}}}
\end{aligned}
$$

into $F_{0}$, it is found that

$$
\begin{aligned}
& F_{0}=\int_{0}^{1} \int_{0}^{1} \int_{0}^{1} \frac{1}{H(X, y, z)}+3 H(X, y, z) \\
& \times\left\{H^{-2}(X, y, z)-\frac{\overline{H^{-2}(X, y, z)^{z}}}{\overline{H^{-3}(X, y, z)^{z}}} H^{-3}(X, y, z)\right. \\
& +\frac{H^{-3}(X, y, z)}{\overline{H^{-3}(X, y, z)^{z}}}\left[\overline{H^{-2}(X, y, z)^{z}}\right. \\
& \left.-\left(\overline{\overline{\overline{H^{-2}(X, y, z)^{z^{y}}}}} \overline{\overline{H^{-3}(X, y, z)^{z^{y}}}}\right) \overline{H^{-3}(X, y, z)^{z}}\right] \\
& \left.+\frac{H^{-3}(X, y, z)}{\overline{H^{-3}(X, y, z)^{z}}} \frac{\overline{H^{-3}(X, y, z)^{z}}}{\overline{\overline{H^{-3}(X, y, z)^{z^{y}}}}} \frac{\mathrm{d} P_{0}}{\mathrm{~d} X}\right\} \mathrm{d} z \mathrm{~d} y \mathrm{~d} X
\end{aligned}
$$

Figure 3 displays the convergence of $F_{\varepsilon}$. Actually, Fig. 3 visualizes the variation with $\varepsilon$ in the expression

$$
\frac{\left|F_{\varepsilon}-F_{0}\right|}{F_{0}}=\frac{\left|\mathcal{F}_{\varepsilon}-\mathcal{F}_{0}\right|}{\mathcal{F}_{0}}
$$

In comparison to the (almost) linear convergence for $\mathcal{I}_{\varepsilon}$, the rate of convergence of $\mathcal{F}_{\varepsilon}$ is lower than linear, according to the figure. However, the results presented above, particularly those shown in Figs 1 and 3, clearly serve as justification of the applicability of the proposed reiterated homogenization result. More specifically, the discrepancies in terms of predicted load carrying capacity and friction force are tolerably small; $\mathcal{O}$ ( 1 per cent) for textures as well as roughness of wavelengths likely to be found in a real application. That is, wavelengths within the ranges $1 / 100-1 / 10$ of the length bearing for the texture and $1 / 10000-1 / 100$ for the roughness.

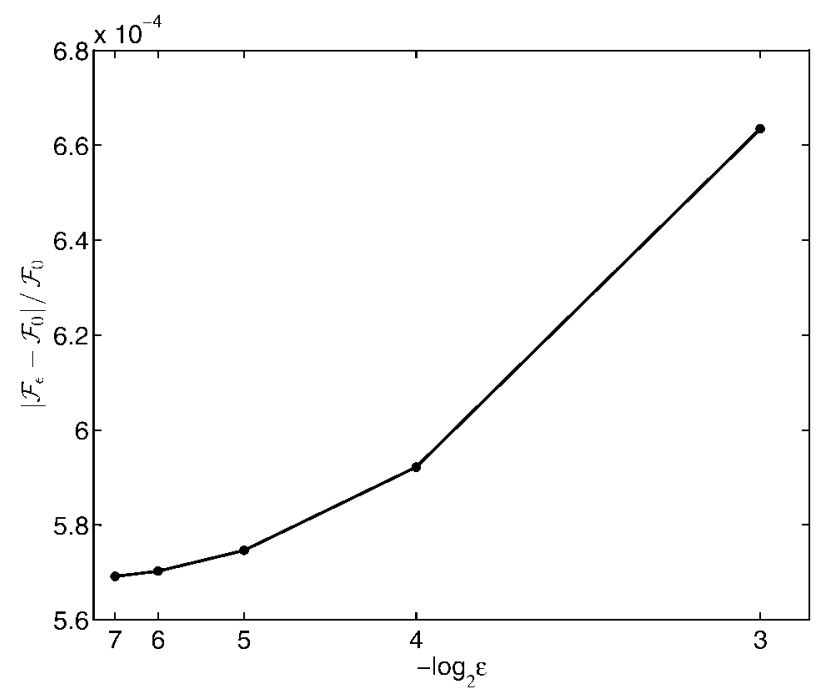

Fig. 3 Convergence of friction force $\mathcal{F}_{\varepsilon}$ towards $\mathcal{F}_{0}$ with decreasing $\varepsilon$. 


\subsection{Application to a thrust pad bearing problem}

The effects of periodic texture and surface roughness are here exemplified by considering a thrust pad bearing problem. The flow is assumed to be modelled through the cylindrical coordinate formulation of the Reynolds problem, i.e. equation (16). A point $x$ in the bearing is identified by its cylindrical coordinates $x=\left(x_{1}, x_{2}\right) \in \Omega=\left[-\theta_{0} / 2, \theta_{0} / 2\right] \times\left[R, 2 R\right.$ (with $x_{1}$ denoting the angular and $x_{2}$ the radial coordinate). In this case, convergence of frictional torque, $\mathcal{T}_{\varepsilon}$, is given by equation (18).

There are two ways of approaching the lubrication problem. In the preceding section, the separation $h_{\text {min }}$ between the surfaces on the global scale is regarded as an input parameter and retrieved the solution in terms of the single dependent parameter, i.e. dimensionless hydrodynamic pressure $P_{\varepsilon}$, by solving the Reynolds equation (20). Observe that due to the specific dimensionless formulation chosen, the solution $p_{\varepsilon}$ for arbitrary $h_{\min }>0$ is obtained.

In approaching the present thrust pad bearing problem, a force-balance equation is employed

$$
W-\int_{\Omega} p_{\varepsilon}(x) \quad x_{2} \mathrm{~d} x_{2} \mathrm{~d} x_{1}=0
$$

where the applied load $W$ appears as an input parameter. The Reynolds equation (26) and the forcebalance equation (26) are then solved to retrieve the solution in terms of the two dependent parameters, namely the separation between the surfaces on the global scale $h_{00}$ and the hydrodynamic pressure $p_{\varepsilon}$. Again, equation (11) is used to represent the film thickness and define

$$
h_{0}(x)=h_{00}-\frac{x_{2}\left(\sin \left(x_{1}\right)-\sin \left(\theta_{0}\right)\right)}{R \sin \left(\theta_{0}\right)} \theta_{0} R \tan \alpha
$$

to model a single bearing segment. Note that $h_{00}$ exactly defines the height of the parallel gap between the trailing edge and the rotating shaft surface and that its value depends on the applied load $W$. (For the smooth problem ( $\left.h_{\mathrm{T}}=h_{\mathrm{R}} \equiv 0\right), h_{00}$ represents the minimum film thickness.) In equation (27), $R$ denotes the inner radius, $\theta_{0}$ defines the size of the pad in radians and $\alpha$ controls the pad inclination. See Fig. 4 for a schematic description of a bearing segment within the bearing.

The effects of periodic texture and surface roughness are examined by considering the homogenized correspondence equations (8) to (16). The case of transversal sinusoidal surface texture as well as the surface roughness is addressed first

$$
\begin{aligned}
h_{\varepsilon}(x)= & h_{00}^{\varepsilon}-\frac{x_{2}\left(\sin \left(x_{1}\right)-\sin \left(\theta_{0}\right)\right)}{R \sin \left(\theta_{0}\right)} \theta_{0} R \tan \alpha \\
& +h_{\mathrm{T}}^{\varepsilon}(x)+h_{\mathrm{R}}^{\varepsilon}(x)
\end{aligned}
$$

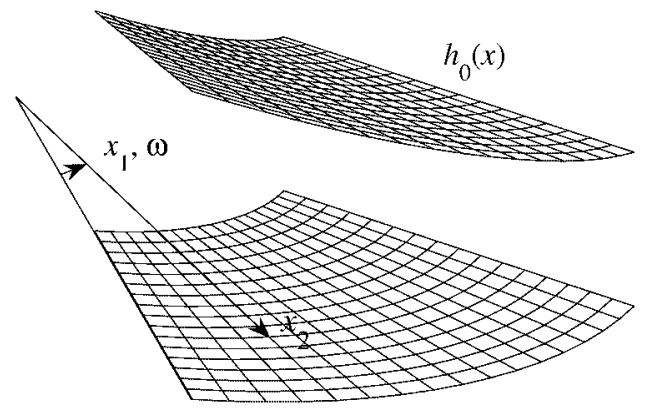

Fig. 4 A schematic descriptions of a single pad

where

$$
h_{\mathrm{T}}^{\varepsilon}(x):=h_{\mathrm{T}}\left(\frac{x}{\varepsilon}\right) \quad \text { and } \quad h_{\mathrm{R}}^{\varepsilon}(x):=h_{\mathrm{R}}\left(\frac{x}{\varepsilon}\right)^{2}
$$

Explicitly, the auxiliary functions are

$$
h_{\mathrm{T}}(y)=\frac{a_{\mathrm{T}}}{2}\left[1-\cos \left(2 \pi y_{1}\right)\right]
$$

and

$$
h_{\mathrm{R}}(z)=\frac{a_{\mathrm{R}}}{2}\left[1-\cos \left(2 \pi z_{1}\right)\right]
$$

The separation $h_{00}^{\varepsilon}$ is regarded as a parameter that is parameterized in $\varepsilon$ and dependent on $W$, and therefore the Reynolds equation (16) and the force-balance criterion (26) are solved for $h_{00}^{\varepsilon}$ and $p_{\varepsilon}$ (or $h_{00}^{0}$ and $p_{0}$ for the corresponding homogenized system of equations). The input parameters chosen for this specific problem are found in Table 1.

To resolve the direct numerical solution (DNS) properly, each roughness wavelength is resolved with $2^{5}$ discrete nodes. For the results presented here, this means a total number of uniformly distributed nodes of $2^{5}\left(2^{4}\right)^{2}=2^{13}$ in the $x_{1}$-direction for $\varepsilon=2^{-4}$, whereas $2^{6}$ nodes were considered sufficient for the discretization in the $x_{2}$-direction. The coefficients in the homogenized matrix and vector, both given in equation (7), were obtained by solving the (one-dimensional) periodic $Y$ or $Z$ cell problems with $2^{6}$ nodes in the $y_{1}$ - and the $z_{1}$-directions.

Table 1 Input parameters

\begin{tabular}{llll}
\hline Parameter & Description & Value & Unit \\
\hline$\theta_{0}$ & ${\text { Pad size (in }{ }^{\circ} \text { ) }}_{R}$ & 25 & $\circ$ \\
$\mu$ & Pad inner radius & $10 \cdot 10^{-3}$ & $\mathrm{~m}$ \\
$\omega$ & Fluid viscosity & 0.3 & $\mathrm{~Pa} \mathrm{~s}$ \\
& Smooth surface & 2.5 & $\mathrm{rad} / \mathrm{s}$ \\
$\alpha$ & $\quad$ angular speed & & \\
$W$ & Pad inclination & $1.6 \cdot 10^{-4}$ & $\mathrm{rad}$ \\
$a$ & Applied load & 10 & $\mathrm{~N}$ \\
& Roughness ampli- & $0.5 \cdot 10^{-6}$ & $\mathrm{~m}$ \\
& fication scaling & & \\
\hline
\end{tabular}


Table 2 Normalized homogenized property $h_{00}^{0} / h_{00}^{\mathrm{s}}$, transversal sinusoidal texture and roughness

\begin{tabular}{llllll}
\hline$a_{\mathrm{T}} \backslash a_{\mathrm{R}}$ & 0 & $a$ & $2 a$ & $4 a$ & $8 a$ \\
\hline 0 & 1.0000 & 0.9639 & 0.9298 & 0.8677 & 0.7636 \\
$a$ & 0.9639 & 0.9278 & 0.8937 & 0.8317 & 0.7277 \\
$2 a$ & 0.9298 & 0.8937 & 0.8597 & 0.7978 & 0.6942 \\
$4 a$ & 0.8677 & 0.8317 & 0.7979 & 0.7363 & 0.6341 \\
$8 a$ & 0.7636 & 0.7277 & 0.6942 & 0.6341 & 0.5364 \\
\hline
\end{tabular}

Table 2 displays normalized homogenized separation $h_{00}^{0} / h_{00}^{\mathrm{s}}$, where $h_{00}^{\mathrm{s}}=6.72 \cdot 10^{-6} \mathrm{~m}$ denotes the minimum film thickness for the correspondingly smooth problem. In the table, texture amplitude $a_{\mathrm{T}}$ increases vertically downwards, whereas roughness amplitude $a_{\mathrm{R}}$ increases horizontally to the right, as indicated by $a_{\mathrm{T}} \backslash a_{\mathrm{R}}$.

For $\varepsilon=2^{-4}$, the maximum relative difference between $h_{00}^{\varepsilon}$ and $h_{00}^{0}$ was found to be less than 0.01 . More specifically, for $\varepsilon=2^{-4}$, corresponding to a texture wavelength $\theta_{0} 2 R / 2^{4} \approx 0.5 \cdot 10^{-3} \mathrm{~m}$ measured at the outer radius $\left(x_{2}=2 R\right)$ and for $\left(a_{\mathrm{T}}, a_{\mathrm{R}}\right)=(8 a, 8 a)$, $\left|h_{00}^{\varepsilon}-h_{00}^{0}\right| / h_{00}^{0}=0.0097$ is obtained. The fact that the maximum difference occurs for $\left(a_{\mathrm{T}}, a_{\mathrm{R}}\right)=(8 a, 8 a)$ is to be expected, as an increase in texture amplitude or roughness amplitude also increases the discretization errors. Since, in theory, it makes sense to distinguish between roughness and texture only when both of them are present, the first row and column in Table 2 could be used as a benchmark of the numerical routine employed. Although the figures in the table seem to indicate that the rigid body separation is symmetrical with respect to texture and roughness amplitude, no theoretical evidence supporting this is reported here.

Table 3 presents the variation in the normalized homogenized frictional torque, $\mathcal{T}_{0} / \mathcal{T}_{\mathrm{s}}$. The numerical values of $\mathcal{T}_{0}$ are computed from equation (18) and the frictional torque exhibited for a set of perfectly smooth surfaces, is found to be $\mathcal{T}_{\mathrm{s}}=1.16 \cdot 10^{-3} \mathrm{Nm}$. Also, for $\varepsilon=2^{-4}$ and $\left(a_{\mathrm{T}}, a_{\mathrm{R}}\right)=(8 a, 8 a)$, it is found that $\left|\mathcal{T}_{\varepsilon}-\mathcal{T}_{0}\right| / \mathcal{T}_{0}=0.0037$.

According to Table 3 , the previously remarked symmetry observed in Table 2, with respect to texture and roughness amplitude also to hold true for the homogenized frictional torque. For example, a texture

Table 3 Normalized homogenized frictional torque $\mathcal{T}_{0} / \mathcal{T}_{\mathrm{s}}$, transversal sinusoidal texture and roughness

\begin{tabular}{llllll}
\hline$a_{\mathrm{T}} \backslash a_{\mathrm{R}}$ & 0 & $a$ & $2 a$ & $4 a$ & $8 a$ \\
\hline 0 & 1.0000 & 1.0005 & 1.0021 & 1.0083 & 1.0312 \\
$a$ & 1.0005 & 1.0011 & 1.0026 & 1.0087 & 1.0314 \\
$2 a$ & 1.0021 & 1.0027 & 1.0043 & 1.0106 & 1.0333 \\
$4 a$ & 1.0083 & 1.0089 & 1.0106 & 1.0170 & 1.0404 \\
$8 a$ & 1.0312 & 1.0318 & 1.0336 & 1.0403 & 1.0641 \\
\hline
\end{tabular}

of amplitude $2 a$ combined with roughness of amplitude 0, i.e. $\left(a_{\mathrm{T}}, a_{\mathrm{R}}\right)=(2 a, 0)$, and a texture of amplitude 0 combined with roughness of amplitude $2 a$, i.e. $\left(a_{\mathrm{T}}, a_{\mathrm{R}}\right)=(0,2 a)$, yields approximately the same $h_{00}^{0}$ or $\mathcal{T}_{0}$ according to the tables, i.e. $h_{00}^{0}=0.9298$ and $\mathcal{T}_{0}=1.0021$, whereas $\left(a_{\mathrm{T}}, a_{\mathrm{R}}\right)=(a, a)$ results in $h_{00}^{0}=0.9278$ and $\mathcal{T}_{0}=1.0011$. However, superpositioning the effects resulting from $\left(a_{\mathrm{T}}, a_{\mathrm{R}}\right)=(a, 0)$ and $\left(a_{\mathrm{T}}, a_{\mathrm{R}}\right)=(0, a)$ gives, with four decimal places, $h_{00}^{0}=$ 0.9278 and $\mathcal{T}_{0}=1.0010$. The relative discrepancies between the superpositioned results and the directly computed results were found to be $3.15 \cdot 10^{-6}$ for $h_{00}^{0}$ and $1.35 \cdot 10^{-5}$ for $\mathcal{T}_{0}$. For the frictional torque, it is suggested that this relative difference is attributed to the last term in equation (18), i.e. the term for the combined effect of texture and roughness.

Next, the textured pad from the preceding case is considered, i.e. equations (28) and (29), but with a longitudinal instead of a transversal sinusoidally shaped surface roughness,

$$
h_{\mathrm{R}}(z)=\frac{a_{\mathrm{R}}}{2}\left[1-\cos \left(2 \pi z_{2}\right)\right]
$$

The results are compiled in Tables 4 and 5 .

These tables illustrate how a longitudinally shaped roughness (or texture, interpreting the data in the first row as being induced by a surface texture instead of surface roughness) influences film formation to a higher degree than the transversal correspondence. When considering the induced frictional torque, the effects caused by the longitudinally shaped roughness (or texture) shows a less pronounced effect than that of the corresponding transversal case. This corresponds well with what would be intuitively expected

Table 4 Normalized homogenized property $h_{00}^{0} / h_{00}^{s}$, transversal sinusoidal texture and longitudinal sinusoidal roughness

\begin{tabular}{llllll}
\hline$a_{\mathrm{T} \backslash a_{\mathrm{R}}}$ & 0 & $a$ & $2 a$ & $4 a$ & $8 a$ \\
\hline 0 & 1.0000 & 0.9627 & 0.9251 & 0.8493 & 0.6947 \\
$a$ & 0.9639 & 0.9266 & 0.8890 & 0.8132 & 0.6585 \\
$2 a$ & 0.9298 & 0.8925 & 0.8549 & 0.7790 & 0.6242 \\
$4 a$ & 0.8677 & 0.8304 & 0.7928 & 0.7167 & 0.5610 \\
$8 a$ & 0.7636 & 0.7262 & 0.6884 & 0.6115 & 0.4532 \\
\hline
\end{tabular}

Table 5 Normalized homogenized frictional torque $\mathcal{T}_{0} / \mathcal{T}_{\mathrm{s}}$, transversal sinusoidal texture and longitudinal sinusoidal roughness

\begin{tabular}{llllll}
\hline$a_{\mathrm{T}} \backslash a_{\mathrm{R}}$ & 0 & $a$ & $2 a$ & $4 a$ & $8 a$ \\
\hline 0 & 1.0000 & 1.0003 & 1.0013 & 1.0053 & 1.0219 \\
$a$ & 1.0005 & 1.0009 & 1.0019 & 1.0059 & 1.0225 \\
$2 a$ & 1.0021 & 1.0025 & 1.0035 & 1.0075 & 1.0242 \\
$4 a$ & 1.0083 & 1.0087 & 1.0098 & 1.0138 & 1.0305 \\
$8 a$ & 1.0312 & 1.0316 & 1.0327 & 1.0369 & 1.0541 \\
\hline
\end{tabular}


Table 6 Normalized homogenized property $h_{00}^{0} / h_{00}^{\mathrm{s}}$, different textures and roughnesses

\begin{tabular}{llllll}
\hline & Smooth & $\begin{array}{l}\text { equation } \\
(30)\end{array}$ & $\begin{array}{l}\text { equation } \\
(31)\end{array}$ & Fig. 7 & Fig. 8 \\
\hline Smooth & 1.0000 & 0.8054 & 0.7615 & 0.5641 & 0.7781 \\
equation (30) & 0.7636 & 0.5752 & 0.5218 & 0.3355 & 0.5464 \\
equation (31) & 0.6947 & 0.5002 & 0.4573 & 0.2596 & 0.4723 \\
equation (32) & 0.9346 & 0.7420 & 0.6951 & 0.5012 & 0.7142 \\
equation (33) & 0.9808 & 0.7866 & 0.7422 & 0.5454 & 0.7592 \\
\hline
\end{tabular}

Table 7 Normalized homogenized property $\mathcal{T}_{0} / \mathcal{T}_{\mathrm{s}}$, different textures and roughnesses

\begin{tabular}{|c|c|c|c|c|c|}
\hline $\mathrm{T}_{\text {Type }} \backslash \mathrm{R}_{\text {Type }}$ & Smooth & $\begin{array}{l}\text { equation } \\
\text { (30) }\end{array}$ & $\begin{array}{l}\text { equation } \\
\text { (31) }\end{array}$ & Fig. 7 & Fig. 8 \\
\hline Smooth & 1.0000 & 1.0199 & 1.0133 & 1.0245 & 1.0197 \\
\hline equation (30) & 1.0312 & 1.0524 & 1.0451 & 1.0573 & 1.0521 \\
\hline equation (31) & 1.0219 & 1.0471 & 1.0370 & 1.0534 & 1.0458 \\
\hline equation (32) & 1.0143 & 1.0349 & 1.0281 & 1.0396 & 1.0347 \\
\hline equation (33) & 1.0027 & 1.0228 & 1.0161 & 1.0274 & 1.0226 \\
\hline
\end{tabular}

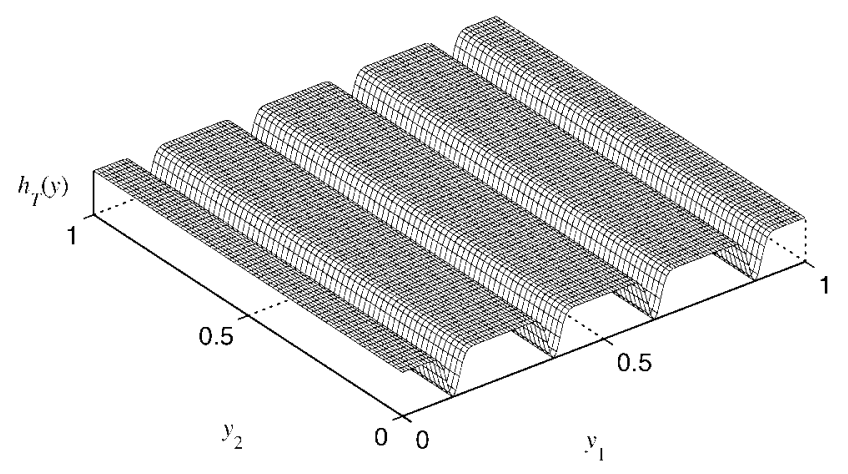

Fig. 5 Artificially ground surface texture $h_{\mathrm{T}}(y)$

and confirms what is already well known within the field.

Tables 6 and 7 compare the two more realistic sur-

face roughness representations found in Figs 7 and 8 with the previously considered sinusoidal representations as well as the smooth case. In addition to the transversal and longitudinal sinusoidal textures, the textures given by equation (32) (displayed in Fig. 5) and equation (33) (displayed in Fig. 6) were also considered. All four roughness representations were scaled to exhibit an average roughness value $R_{\mathrm{a}}\left(=\int_{Z}\left|h_{\mathrm{R}}(z)-\int_{Z} h_{\mathrm{R}}(z) \mathrm{d} z\right| \mathrm{d} z\right)$ of $1 \mu \mathrm{m}$. This means that the corresponding amplitude of the sinusoidal representations (both the transversal and the longitudinal) become $a_{\mathrm{R}}=R_{z} / 2=\pi \mu \mathrm{m}$, i.e. $R_{z}=2 \pi \mu \mathrm{m} \approx$ $6.28 \mu \mathrm{m}$. The rough surface in the Fig. 7 has $R_{z}=$ $6.10 \mu \mathrm{m}$ and the one in Fig. 8 has $R_{z}=11.00 \mu \mathrm{m}$. In all simulations (except for the case without any texture), the texture amplitude was held fixed, i.e. $a_{\mathrm{T}}=4 \mu \mathrm{m}$.

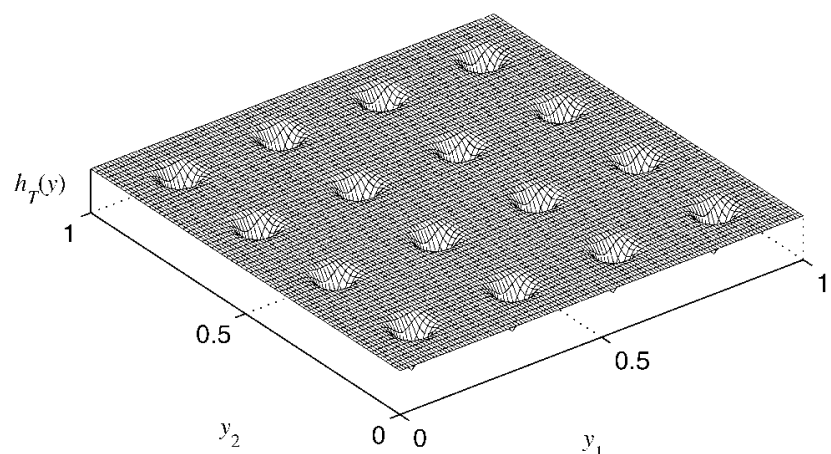

Fig. 6 Artificially dimpled surface texture $h_{\mathrm{T}}(y)$

Figure 5 presents the mathematical description of the surface texture given by

$$
h_{\mathrm{T}}(y)=10^{-50\left(y_{1}-1 / 2\right)^{2}} \cos \left[2 \pi\left(y_{1}-\frac{1}{2}\right)\right]
$$

while the surface representation presented in Fig. 6 is modelled mathematically by

$$
\begin{aligned}
h_{\mathrm{T}}(y)= & 10^{-25\left(\left(y_{1}-1 / 2\right)^{2}+\left(y_{2}-1 / 2\right)^{2}\right)} \cos \left[2 \pi\left(y_{1}-\frac{1}{2}\right)\right] \\
& \times \cos \left[2 \pi\left(y_{2}-\frac{1}{2}\right)\right]
\end{aligned}
$$

Figure 7 displays a surface roughness representation $h_{\mathrm{R}}(z)$, exhibiting an almost unskewed striated pattern, whereas Fig. 8 displays a negatively skewed surface roughness representation $h_{\mathrm{R}}(z)$ that exhibits a reasonably random pattern. Both of these roughnesses originate from measurements but have been resampled and normalized for the assessments conducted here. Normalized to an average roughness value, $R_{\mathrm{a}}=1 \mu \mathrm{m}$, these roughness representations have $R_{z}=6.10 \mu \mathrm{m}$ and $R_{z}=11.00 \mu \mathrm{m}$ (as previously mentioned) and their corresponding skewness values, $R_{\mathrm{SK}}=-0.0061$ and $R_{\mathrm{SK}}=-1.7284$. In studying Table 6 , one notices that the longitudinal texture deteriorates film formation most, i.e. produces the smallest values of the ratio $h_{00}^{0} / h_{00}^{\mathrm{s}}$, and the artificially dimpled texture (33) the least without considering the perfectly

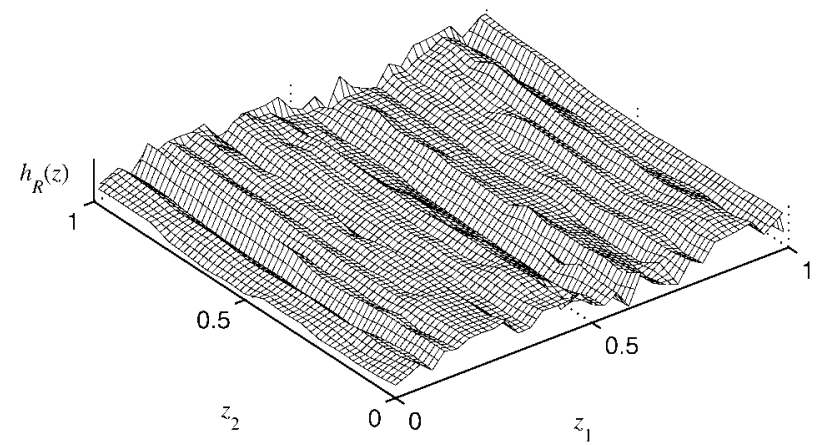

Fig. 7 A surface roughness $h_{\mathrm{R}}(z)$, exhibiting a striated pattern, originating from a surface measurement 


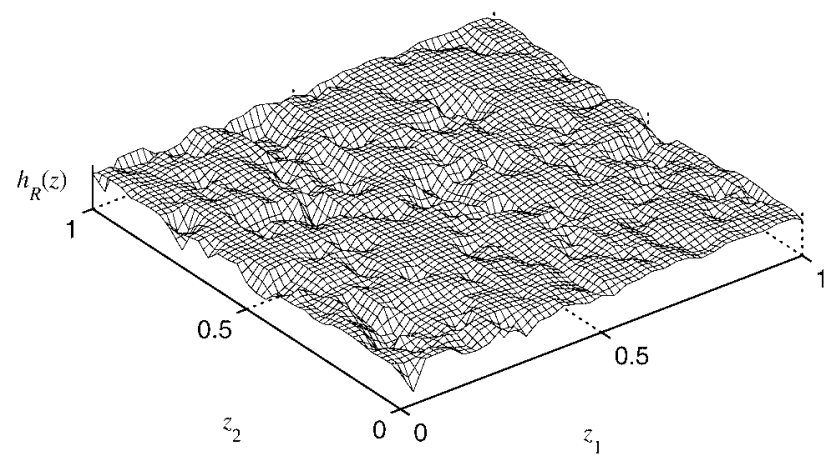

Fig. 8 A negatively skewed surface roughness $h_{\mathrm{R}}(z)$ exhibiting a reasonably random pattern, originating from a surface measurement

smooth surface. The surface roughness representation shown in Fig. 7 is by far the most detrimental in terms of film formation. This surface roughness representation exhibits exactly the same $R_{\mathrm{a}}$ (ensured by the scaling) and approximately the same $R_{z}$ and $R_{\mathrm{SK}}$ values as those corresponding to the transversal sinusoidal representation. The same table clearly shows that after the perfectly smooth surfaces, it is the transversal sinusoidal roughness representation that generates the thickest film. Hence, it is concluded that for a prediction to be reliable it must consider more information than the three abovementioned surface roughness parameters.

Addressing frictional torque, it is - according to Table 7 - the artificially dimpled texture (33) is again the texture inducing the smallest effect. However, it is the transversal and not the longitudinal sinusoidal texture that influences frictional torque the most. In optimizing the performance in terms of film formation and induced frictional torque, it is clear that perfectly smooth surfaces are preferred, this was also previously confirmed, see e.g. [3]. However, disregarding the unrealistic perfectly smooth bearing, it is the artificially dimpled surface texture (33) that yields the thickest films and induces the smallest frictional torque. As well, it is the grounded surface roughness representation displayed in Fig. 7 that clearly has the most severe influence on film formation and frictional torque. Thus, from a manufacturing point-of-view, in choosing from the selection of textures and roughnesses found in Tables 6 and 7, it would probably be most convenient to use a laser dimpling technique to achieve the $4 \mu \mathrm{m}$ deep texture and then radially grind to a $1 \mu \mathrm{m} R_{\mathrm{a}}$-value. This would be a rather successful combination according to the present findings. However, if the surface is further processed from its grounded state, e.g. also chemically de-burred, it might display a surface finish similar to that presented in Fig. 8. In turn, this should facilitate film formation as well as lower the induced frictional torque, according to the results presented here.

\section{CONCLUSIONS}

The main result is that a reiterated homogenization procedure is successfully developed for a class of problems by using multiple scale expansion. In particular, the Reynolds problem, which governs incompressible and Newtonian flow in Cartesian and cylindrical coordinates, belongs to this class. This made it possible to efficiently study problems connected to hydrodynamic lubrication including shape, texture and roughness. Herein lies the novelty of the results, whereas only two scales, i.e. shape and roughness, have been considered previously we can consider a third scale, i.e. the texture.

In addition, the convergence of the pressure gradient is analysed. This enabled to study the limiting behaviour of hydrodynamically induced friction force and frictional torque, as the wavelengths of the local scales tend to zero.

To demonstrate the applicability and effectiveness of the method, several numerical results are presented, which clearly show the convergence of the deterministic solutions towards the homogenized solution. The quantification of convergence was given in terms of load carrying capacity and friction force. In these convergence illustrations, only transversal and longitudinal roughness and texture were considered. The reason for this was that it is impossible to obtain the full numerical solution for two-dimensional roughness and texture, due to enormous amount of discretization points, which are required to resolve the surface. However, by using the homogenization result it is possible to study the effects of arbitrary roughness with very high accuracy by solving the derived smooth homogenized equation. This was demonstrated in an example connected to a realistic thrust pad bearing problem, where the effects of texture and roughness on film formation and frictional torque were investigated.

Based on the general convergence result for the pressure gradient, the limit of the deterministic expression is deduced for the frictional force. The resulting homogenized quantity is made up of friction force due to the smooth (averaged) film thickness plus a corrector term. Moreover, in this corrector term, one can identify three separate contributions, i.e. due to either roughness or texture acting alone or texture and roughness acting together. The presence of terms of the latter kind implies that roughness could enhance (or diminish) certain effects that are essentially due to texture (and vice versa). The numerical results indicate that the combined effect due to texture and roughness on the modelled hydrodynamic bearings can be efficiently analysed using reiterated homogenization. The resulting discrepancies in terms of predicted load carrying capacity and friction force are small; $\mathcal{O}(1$ per cent) for textures as well as roughnesses of wavelengths likely to be found in a real application. 
That is, wavelengths within the ranges $1 / 100-1 / 10$ of the length bearing for the texture and $1 / 10000-1 / 100$ for roughness.

From the assessment of the combined effects of texture and roughness - that arise in the modelled thrust pad bearing - the conclusion that reiterated homogenization is a feasible tool is drawn. For any prediction to be reliable, more information regarding the surface than the three surface roughness parameters, $R_{\mathrm{a}}, R_{z}$, and $R_{\mathrm{SK}}$ are required.

\section{ACKNOWLEDGEMENTS}

This work was partly financed by the European Commission, Marie Curie Transfer of Knowledge Scheme Predicting Lubricant Performance for Improved Efficiency (FP6), the Swedish Research Council, An interdisciplinary study of rough surface effects in lubrication by homogenization techniques, 621-2005-3168, and Ghana Government Scholarship Secretariat.

\section{REFERENCES}

1 Bayada, G. and Faure, J. B. A double scale analysis approach of the Reynolds roughness comments and application to the journal bearing. J. Tribol., 1989, 111 (3), 323-330.

2 Bayada, G., Martin, S., and Vazquez, C. An average flow model of the Reynolds roughness including a mass-flow preserving cavitation model. J. Tribol., 2005, 127(4), 793802.

3 Buscaglia, G. C., Ciuperca, I., and Jai, M. The effect of periodic textures on the static characteristics of thrust bearings. J. Tribol., 2005, 127(4), 899-902.

4 Almqvist, A. and Dasht, J. The homogenization process of the Reynolds equation describing compressible liquid flow. J. Tribol., 2006, 39, 994-1002.

5 Kane, M. and Bou-Said, B. Comparison of homogenization and direct techniques for the treatment of roughness in incompressible lubrication. J. Tribol., 2004, 126, 733-737.

6 Kane, M. and Bou-Said, B. A study of roughness and nonNewtonian effects in lubricated contacts. J. Tribol., 2005, 127, 575-581.

7 Almqvist, A., Essel, E. K., Persson, L.-E., and Wall, P. Homogenization of the unstationary incompressible Reynolds equation. Tribol. Int., 2007, 40, 1344-1350.

8 Bensousan, A., Lions, J.-L., and Papanicolaou, G. Asymptotic analysis for periodic structures, NorthHolland, Amsterdam-New-York-Oxford, 1978.

9 Lions, J.-L., Lukkassen, D., Persson, L.-E., and Wall, P. Reiterated homogenization of nonlinear monotone operators. Chin. Ann. Math. Ser. B., 2001, 22, 1-12.

10 Allaire, G. and Briane, M. Multiscale convergence and reiterated homogenization. Proc. R. Soc. Edinb., 1996, 126, 297-342.

11 Persson, L.-E., Persson, L., Svanstedt, N., and Wyller, J. The homogenization method: an introduction, 1993 (Studentlitteratur, Lund).
12 Almqvist, A., Larsson, R., and Wall, P. The homogenization process of the time dependent Reynolds equation describing compressible liquid flow. Tribologia - Finnish J. Tribol., 2007, 26, 30-44.

13 Lions, J. L., Lukkassen, D., Persson, L. E., and Wall, P. Reiterated homogenization of nonlinear monotone operators. Chin. Ann. of Math., 2001, 22B(1), 1-12.

14 Lukkassen, D., Nguetseng, G., and Wall, P. Two scale convergence. Int. IJPAM., 2002, 2(1), 35-86.

\section{BIBLIOGRAPHY}

Cioranescu, D. and Donato, P. An introduction to homogenization, Oxford Lecture Series in Mathematics and its Applications, 1999 (Oxford University Press, New York).

\section{APPENDIX 1}

\section{Notation}

$a_{i j} \quad$ elements of matrix $\mathbf{A}$

$\mathbf{A}_{\varepsilon} \quad$ deterministic matrix

$\mathbf{A}_{0} \quad$ homogenized matrix

$A_{i} \quad$ differential operator, $i=0, \ldots, 4$

$\begin{array}{ll}\boldsymbol{b}_{\varepsilon} & \text { deterministic vector }\end{array}$

$\boldsymbol{b}_{0} \quad$ homogenized vector

$e_{i} \quad$ Canonical basis in $\mathbb{R}^{2}, e_{1}=(1,0)$ and

$e_{2}=(0,1)$

$\bar{f}^{z} \quad$ average of $f$ with respect to $Z\left(=\int_{Z} f \mathrm{~d} z\right)$

$\overline{\bar{f}}^{z}$

average of $f$ with respect to $Z$ and

$Y\left(=\int_{Y} \int_{Z} f \mathrm{~d} z \mathrm{~d} y\right)$

$\mathcal{F}_{\varepsilon} \quad$ deterministic frictional force

$\mathcal{F}_{0} \quad$ homogenized frictional force

$F_{\varepsilon} \quad$ dimensionless friction force

$\left.=\mathcal{F}_{\varepsilon} / \mu U L / h_{\min }\right)$

$F_{0} \quad$ dimensionless homogenized friction force

$h \quad$ auxiliary function used to model film thickness

$h_{0} \quad$ function describing global geometry of bearing

$h_{\mathrm{R}} \quad$ Function describing the roughness part of film thickness

$h_{\varepsilon} \quad$ deterministic film thickness

$h_{\mathrm{T}} \quad$ function describing the texture part of film thickness

$h_{\text {min }} \quad$ fixed minimum film thickness $=\min$ of $h_{0}$

$H \quad$ dimensionless film thickness $=h / h_{\text {min }}$

$\mathcal{I}_{\varepsilon} \quad$ deterministic load carrying capacity

$\mathcal{I}_{0} \quad$ homogenized load carrying capacity

$L \quad$ Length of stationary surface exhibiting texture and roughness

$p_{\varepsilon} \quad$ deterministic pressure solution

$p_{i} \quad$ the $i$ th term in the expansion of the pressure $p_{\varepsilon}$

$p_{0} \quad$ homogenized pressure solution 
$P_{\varepsilon} \quad$ dimensionless deterministic

pressure $=p_{\varepsilon} /\left(6 \mu U L / h_{\min }^{2}\right)$

$\mathcal{T}_{\varepsilon} \quad$ deterministic frictional torque

$\mathcal{T}_{0} \quad$ homogenized frictional torque

$\mathcal{T}_{\mathrm{s}} \quad$ frictional torque for a perfectly smooth

surface $\left(=1.16 \cdot 10^{-3} \mathrm{Nm}\right)$

$u_{i} \quad Z$-periodic solution of the local problems, $i=0,1,2$

$U \quad$ linear speed of moving surface

$v_{i} \quad Y$-periodic solution of the local problems, $i=0,1,2$

$x \quad$ local spatial coordinate, $x=\left(x_{1}, x_{2}\right)$

$X \quad$ dimensionless spacial coordinate $=x / L$

$y \quad$ local spatial coordinate,

$y=\left(y_{1}, y_{2}\right)=\left(x_{1} / \varepsilon, x_{2} / \varepsilon\right)$

$Y \quad Y$-cell $=[0,1]^{2}$

$z \quad$ local spatial coordinate,

$z=\left(z_{1}, z_{2}\right)=\left(x_{1} / \varepsilon^{2}, x_{2} / \varepsilon^{2}\right)$

$Z \quad Z$-cell $=[0,1]^{2}$

$\varepsilon \quad$ parameter describing the roughness and texture scale $(\varepsilon>0)$

$\Omega \quad$ open bounded subset of $\mathbb{R}^{2}$

$\partial \Omega \quad$ Boundary of $\Omega$

$\nabla_{x} \quad$ gradient operator, $\nabla_{x}=\nabla$

$\nabla_{y} \quad$ Gradient operator, $\nabla_{y}=\left(\partial / \partial y_{1}, \partial / \partial y_{2}\right)$

$\nabla_{z} \quad$ gradient operator, $\nabla_{z}=\left(\partial / \partial z_{1}, \partial / \partial z_{2}\right)$

\section{APPENDIX 2}

In this appendix the analysis leading to the homogenization result is presented, by deriving the homogenized equation (8) corresponding to equation (1). The method used is known as multiple scale expansion. For more information concerning this method in connection with homogenization see e.g. [8].

It is observed that the chain rule applied to a smooth function of the form $\psi_{\varepsilon}(x)=\psi(x, y, z)$, where $y=x / \varepsilon$ and $z=x / \varepsilon^{2}$ gives that

$$
\nabla_{x} \psi_{\varepsilon}(x)=\left(\nabla_{x}+\frac{1}{\varepsilon} \nabla_{y}+\frac{1}{\varepsilon^{2}} \nabla_{z}\right) \psi(x, y, z)
$$

Inserting the expansion equation (2) (of $p_{\varepsilon}$ ) into equation (1) and making use of equation (34), it is obtained

$$
\begin{gathered}
\left(\nabla_{x}+\frac{1}{\varepsilon} \nabla_{y}+\frac{1}{\varepsilon^{2}} \nabla_{z}\right) \cdot\left[\mathbf{A}\left(\nabla_{x}+\frac{1}{\varepsilon} \nabla_{y}+\frac{1}{\varepsilon^{2}} \nabla_{z}\right)\right. \\
\left.\times \sum_{i=0}^{\infty} \varepsilon^{i} p_{i}\right]=\left(\nabla_{x}+\frac{1}{\varepsilon} \nabla_{y}+\frac{1}{\varepsilon^{2}} \nabla_{z}\right) \cdot \boldsymbol{b}
\end{gathered}
$$

Let the differential operators $A_{i}, i=0, \ldots, 4$ be defined as

$$
\begin{aligned}
& A_{0}=\nabla_{z} \cdot\left(\mathbf{A} \nabla_{z}\right) \\
& A_{1}=\nabla_{z} \cdot\left(\mathbf{A} \nabla_{y}\right)+\nabla_{y} \cdot\left(\mathbf{A} \nabla_{z}\right)
\end{aligned}
$$

$$
\begin{aligned}
& A_{2}=\nabla_{x} \cdot\left(\mathbf{A} \nabla_{z}+\nabla_{y} \cdot\left(\mathbf{A} \nabla_{y}\right)+\nabla_{z} \cdot\left(\mathbf{A} \nabla_{x}\right)\right. \\
& A_{3}=\nabla_{x} \cdot\left(\mathbf{A} \nabla_{y}\right)+\nabla_{y} \cdot\left(\mathbf{A} \nabla_{x}\right) \\
& A_{4}=\nabla_{x} \cdot\left(\mathbf{A} \nabla_{x}\right)
\end{aligned}
$$

Using the above notation (35) may be written as

$$
\begin{gathered}
\left(\varepsilon^{-4} A_{0}+\varepsilon^{-3} A_{1}+\varepsilon^{-2} A_{2}+\varepsilon^{-1} A_{3}+A_{4}\right)\left(p_{0}+\varepsilon p_{1}\right. \\
\left.\quad+\varepsilon^{2} p_{2}+\cdots\right)=\left(\varepsilon^{-2} \nabla_{z}+\varepsilon^{-1} \nabla_{y}+\nabla_{x}\right) \cdot \boldsymbol{b}
\end{gathered}
$$

By comparing terms with the same order of $\varepsilon$ (from -4 to 0 ), the following system of equations are obtained

$$
\begin{aligned}
& A_{0} p_{0}=0 \\
& A_{0} p_{1}+A_{1} p_{0}=0 \\
& A_{0} p_{2}+A_{1} p_{1}+A_{2} p_{0}=\nabla_{z} \cdot \boldsymbol{b} \\
& A_{0} p_{3}+A_{1} p_{2}+A_{2} p_{1}+A_{3} p_{0}=\nabla_{y} \cdot \boldsymbol{b} \\
& A_{0} p_{4}+A_{1} p_{3}+A_{2} p_{2}+A_{3} p_{1}+A_{4} p_{0}=\nabla_{x} \cdot \boldsymbol{b}
\end{aligned}
$$

In the following, the following well-known result is frequently used

$$
\Gamma u=f \text { has a solution if and only if } \int_{Z} f \mathrm{~d} z=0
$$

In this case, $u$ is unique up to an additive constant, where $\Gamma$ is any of the operators $A_{0}, A_{1}, A_{2}, \ldots$ and $Z$ may be replaced with $Y$. See for example [11, p. 39] for a proof of equation (37). According to equation (37), it is clear that $p_{0}$ in equation (36a) does not depend on $z$, that is

$$
p_{0}=p_{0}(x, y)
$$

and this simplifies equation (36) to

$$
A_{0} p_{1}(x, y, z)=-\nabla_{z} \cdot\left(\mathbf{A}(x, y, z) \nabla_{y} p_{0}(x, y)\right)
$$

By linearity

$$
\begin{aligned}
p_{1}(x, y, z)= & u_{1}(x, y, z) \frac{\partial p_{0}}{\partial y_{1}}(x, y) \\
& +u_{2}(x, y, z) \frac{\partial p_{0}}{\partial y_{2}}(x, y)+\tilde{p}_{1}(x, y)
\end{aligned}
$$

where the $Z$-periodic function $u_{i}=u_{i}(x, y, z), i=1,2$ is a solution (unique up to a constant) to the following local problem

$$
\nabla_{z} \cdot\left(\mathbf{A}\left(\nabla_{z} u_{i}+\mathbf{e}_{i}\right)\right)=0 \text { in } Z
$$

According to equation (37), (36c) can be solved for $p_{2}$ if and only if

$$
\int_{Z}\left(A_{1} p_{1}+A_{2} p_{0}\right) \mathrm{d} z=0
$$


Substituting equation (40) into equation (42) and considering $Z$-periodicity, it is found that

$$
\nabla_{y} \cdot\left\{\int_{Z}\left[\mathbf{A}\left(\nabla_{y} p_{0}+\nabla_{z}\left(u_{1} \frac{\partial p_{0}}{\partial y_{1}}+u_{2} \frac{\partial p_{0}}{\partial y_{2}}\right)\right)\right] \mathrm{d} z\right\}=0
$$

This is identical to

$$
\nabla_{y} \cdot\left(\overline{\mathbf{A}(x, y, z) \mathcal{A}(x, y, z)^{z}} \nabla_{y} p_{0}(x, y)\right)=0
$$

where $\bar{f}^{z}=\int_{Z} f \mathrm{~d} z$ and

$$
\mathcal{A}=\mathcal{A}(x, y, z)=\left(\begin{array}{cc}
1+\frac{\partial u_{1}}{\partial z_{1}} & \frac{\partial u_{2}}{\partial z_{1}} \\
\frac{\partial u_{1}}{\partial z_{2}} & 1+\frac{\partial u_{2}}{\partial z_{2}}
\end{array}\right)
$$

It is remarked that the equation (44) is the homogenized equation after the first reiteration.

Equation (44) implies that

$$
p_{0}(x, y)=p_{0}(x)
$$

Thus, by virtue of equation (40)

$$
p_{1}=p_{1}(x, y)
$$

Using equations (46) and (47) in equation (36c) and simplifying gives

$$
A_{0} p_{2}=\nabla_{z} \cdot \boldsymbol{b}-\nabla_{z} \cdot\left[\mathbf{A}\left(\nabla_{y} p_{1}+\nabla_{x} p_{0}\right)\right]
$$

By linearity, it is found that $p_{2}$ is of the form

$$
\begin{aligned}
p_{2}(x, y, z)= & u_{0}(x, y, z)+u_{1}(x, y, z) \\
& \left(\frac{\partial p_{0}}{\partial x_{1}}(x)+\frac{\partial p_{1}}{\partial y_{1}}(x, y)\right)+u_{2}(x, y, z) \\
& \left(\frac{\partial p_{0}}{\partial x_{2}}(x)+\frac{\partial p_{1}}{\partial y_{2}}(x, y)\right)+\tilde{p}_{2}(x, y)
\end{aligned}
$$

where $u_{0}$ is a solution (unique up to an additive constant) to the local problem

$$
\nabla_{z} \cdot\left(\mathbf{A} \nabla_{z} u_{0}-\boldsymbol{b}\right)=0 \quad \text { in } Z
$$

Recall that even though $y$ is a parameter in this context, $u_{0}$ in equation (50), and $u_{1}$ and $u_{2}$ in equation (41) are not only $Z$-periodic, but also $Y$-periodic functions.
To solve equation ( $36 \mathrm{~d}$ ) for $p_{3}$, it must hold that

$$
\int_{Z}\left(A_{1} p_{2}+A_{2} p_{1}+A_{3} p_{0}-\nabla_{y} \cdot \boldsymbol{b}\right) \mathrm{d} z=0
$$

Expansion yields

$$
\begin{aligned}
& \int_{Z}\left(\nabla_{y} \cdot\left(\mathbf{A} \nabla_{z} p_{2}\right)+\nabla_{y} \cdot\left(\mathbf{A} \nabla_{y} p_{1}\right)+\nabla_{y} \cdot\left(\mathbf{A} \nabla_{x} p_{0}\right)\right. \\
& \left.\quad-\nabla_{y} \cdot \boldsymbol{b}\right) \mathrm{d} z=0
\end{aligned}
$$

Inserting equation (49) in equation (51) and rearranging the terms, it is obtained that

$$
\begin{aligned}
\int_{Z} \nabla_{y} \cdot\left\{\mathbf{A}\left[\nabla_{x} p_{0}+\nabla_{z}\left(u_{1} \frac{\partial p_{0}}{\partial x_{1}}+u_{2} \frac{\partial p_{0}}{\partial x_{2}}\right)\right]\right. \\
+\left(\mathbf{A} \nabla_{z} u_{0}-\boldsymbol{b}\right)+\mathbf{A}\left[\nabla_{y} p_{1}+\nabla_{z}\left(u_{1} \frac{\partial p_{1}}{\partial y_{1}}\right.\right. \\
\left.\left.\left.+u_{2} \frac{\partial p_{1}}{\partial y_{2}}\right)\right]\right\} \mathrm{d} z=0
\end{aligned}
$$

and by virtue of equation (45), this reduces to

$$
\begin{aligned}
\nabla_{y} \cdot\left(\overline{\mathbf{A} \mathcal{A}}^{z} \nabla_{y} p_{1}\right)= & -\nabla_{y} \cdot\left(\overline{\mathbf{A} \mathcal{A}}^{z} \nabla_{x} p_{0}\right) \\
& +\nabla_{y} \cdot \overline{\left(\boldsymbol{b}-\mathbf{A} \nabla_{z} u_{0}\right)}
\end{aligned}
$$

By linearity, the equation (52) is satisfied if $p_{1}$ is of the form

$$
\begin{aligned}
p_{1}(x, y)= & v_{0}(x, y)+v_{1}(x, y) \frac{\partial p_{0}}{\partial x_{1}}(x) \\
& +v_{2}(x, y) \frac{\partial p_{0}}{\partial x_{2}}(x)+\tilde{p}_{1}(x)
\end{aligned}
$$

where the $Y$-periodic functions $v_{i}=v_{i}(x, y) \quad(i=$ $0,1,2)$ are the solutions of the following local problems involving $y$

$$
\left\{\begin{array}{l}
\nabla_{y} \cdot\left[\overline{\mathbf{A} \mathcal{A}}^{z} \nabla_{y} v_{0}-\left({\overline{\boldsymbol{b}-\mathrm{A} \nabla_{z} u_{0}}}^{z}\right)\right]=0 \quad \text { in } Y \\
\nabla_{y} \cdot\left[\overline{\mathbf{A} \mathcal{A}}^{z}\left(\nabla_{y} v_{i}+e_{i}\right)\right]=0 \text { on } Y, \quad(i=1,2)
\end{array}\right.
$$

Here $x$ is regarded as a parameter.

A necessary condition for solving equation (36e) for $p_{4}$ is that

$$
\int_{Z} A_{1} p_{3}+A_{2} p_{2}+A_{3} p_{1}+A_{4} p_{0}-\nabla_{x} \cdot \boldsymbol{b} \mathrm{d} z=0
$$

By integrating equation (55) over $Y$, expanding the differential operators $A_{i}$ and making use of the $Y$ and $Z$ periodicity yields

$$
\int_{Y} \int_{Z} \nabla_{x} \cdot\left(\mathbf{A}\left(\nabla_{z} p_{2}+\nabla_{y} p_{1}+\nabla_{x} p_{0}\right)-\boldsymbol{b}\right) \mathrm{d} y \mathrm{~d} z=0
$$


Next, it is shown on that the condition (56) leads to the homogenized equation. By inserting equations (49) and (53) in equation (56), it is obtained that

$$
\begin{aligned}
\nabla_{x} \cdot\left\{\overline{\overline{\mathbf{A} \nabla_{z}\left[u_{0}+u_{1}\left(\frac{\partial p_{0}}{\partial x_{1}}+\frac{\partial p_{1}}{\partial y_{1}}\right)\right.}}\right. \\
\quad+\overline{\left.\overline{\left.u_{2}\left(\frac{\partial p_{0}}{\partial x_{2}}+\frac{\partial p_{1}}{\partial y_{2}}\right)+\tilde{p}_{2}(x, y)\right]^{z^{y}}}\right\}} \\
+\nabla_{x} \cdot\left[\overline{\overline{\mathbf{A} \nabla_{y}\left(v_{0}+v_{1} \frac{\partial p_{0}}{\partial x_{1}}+v_{2} \frac{\partial p_{0}}{\partial x_{2}}+\tilde{p}_{1}(x)\right)^{z y}}}\right] \\
+\nabla_{x} \cdot\left(\overline{\overline{\mathbf{A}}}^{y} \nabla_{x} p_{0}\right) \\
=\nabla_{x} \cdot\left(\overline{\overline{\boldsymbol{b}}}^{y}\right)
\end{aligned}
$$

By simplifying and rearranging the following, it is obtained

$$
\begin{aligned}
& \nabla_{x} \cdot\left\{\overline{\mathbf{A}\left[\nabla_{x} p_{0}+\nabla_{y}\left(v_{1} \frac{\partial p_{0}}{\partial x_{1}}+v_{2} \frac{\partial p_{0}}{\partial x_{2}}\right)\right]^{z^{y}}}\right\} \\
& +\nabla_{x} \cdot\left[\overline{\overline{\mathbf{A} \nabla_{z}\left(u_{1} \frac{\partial p_{0}}{\partial x_{1}}+u_{2} \frac{\partial p_{0}}{\partial x_{2}}\right)^{z^{y}}}}\right] \\
& +\nabla_{x} \cdot\left\{\overline{\overline{\mathbf{A} \nabla_{z}\left[u_{1} \frac{\partial}{\partial y_{1}}\left(v_{1} \frac{\partial p_{0}}{\partial x_{1}}+v_{2} \frac{\partial p_{0}}{\partial x_{2}}\right)\right]^{z^{y}}}}\right\} \\
& +\nabla_{x} \cdot\left\{\overline{\mathbf{A} \nabla_{z}\left[u_{2} \frac{\partial}{\partial y_{2}}\left(v_{1} \frac{\partial p_{0}}{\partial x_{1}}+v_{2} \frac{\partial p_{0}}{\partial x_{2}}\right)\right]^{z^{y}}}\right\} \\
& =\nabla_{x} \cdot\left\{\overline{\overline{\boldsymbol{b}-\mathbf{A}\left[\nabla_{z} u_{0}+\nabla_{y} v_{0}+\nabla_{z}\left(u_{1} \frac{\partial v_{0}}{\partial y_{1}}\right)\right.}}\right. \\
& \left.\overline{\overline{\left.+\nabla_{z}\left(u_{2} \frac{\partial v_{0}}{\partial y_{2}}\right)\right]^{z^{y}}}}\right\}
\end{aligned}
$$

By defining

$$
\mathcal{B}=\mathcal{B}(x, y)=\left(\begin{array}{cc}
1+\frac{\partial v_{1}}{\partial y_{1}} & \frac{\partial v_{2}}{\partial y_{1}} \\
\frac{\partial \nu_{1}}{\partial y_{2}} & 1+\frac{\partial v_{2}}{\partial y_{2}}
\end{array}\right)
$$

It is seen that the compressed form of

$$
\begin{aligned}
\nabla_{x} & \cdot\left\{\mathbf{A}\left[\nabla_{x} p_{0}+\nabla_{y}\left(v_{1} \frac{\partial p_{0}}{\partial x_{1}}+v_{2} \frac{\partial p_{0}}{\partial x_{2}}\right)\right]\right\} \\
& =\nabla_{x} \cdot\left(\mathbf{A} \mathcal{B} \nabla_{x} p_{0}\right)
\end{aligned}
$$

Inserting equation (59) into equation (57) and rearranging the terms, it is found that

$$
\begin{aligned}
& \nabla_{x} \cdot\left\{\overline{\overline{\mathbf{A}}}^{z} \nabla_{x} p_{0}\right\}
\end{aligned}
$$

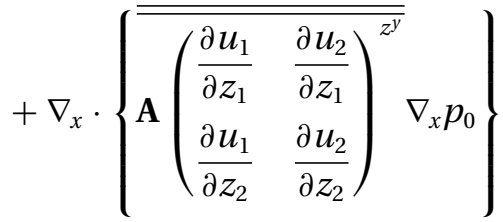

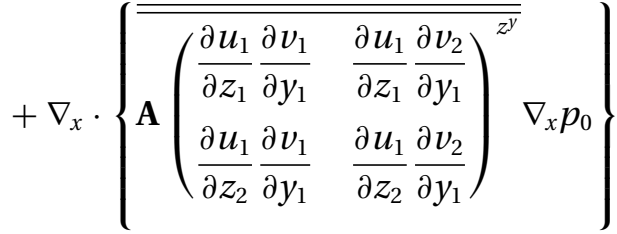

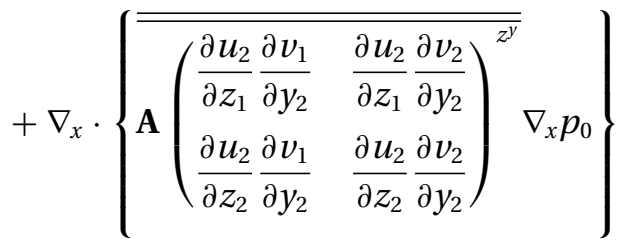

$$
\begin{aligned}
& =\nabla_{x} \cdot\left\{\overline{\overline{\boldsymbol{b}-\mathbf{A}\left[\nabla_{z} u_{0}+\nabla_{y} \nu_{0}+\nabla_{z}\left(u_{1} \frac{\partial v_{0}}{\partial y_{1}}\right)\right.}}\right. \\
& \left.\overline{\overline{\left.+\nabla_{z}\left(u_{2} \frac{\partial v_{0}}{\partial y_{2}}\right]\right)^{z^{y}}}}\right\}
\end{aligned}
$$

By adding the corresponding components of the matrices in the inner brackets and simplifying, it is obtained that

$$
\begin{aligned}
\nabla_{x} \cdot\left({\overline{\overline{\mathbf{A} \mathcal{A B}^{z}}}}^{y} \nabla_{x} p_{0}\right) \\
=\nabla_{x} \cdot\left[\overline{\overline{\boldsymbol{b}-\mathbf{A} \nabla_{z} u_{0}-\mathbf{A}\left(\nabla_{y} v_{0}+\nabla_{z} u_{1} \frac{\partial v_{0}}{\partial y_{1}}\right.}}\right. \\
\left.=\frac{\overline{=}}{\left.+\nabla_{z} u_{2} \frac{\partial \nu_{0}}{\partial y_{2}}\right)^{z^{y}}}\right]
\end{aligned}
$$

Moreover

$$
\nabla_{y} v_{0}+\nabla_{z} u_{1} \frac{\partial v_{0}}{\partial y_{1}}+\nabla_{z} u_{2} \frac{\partial v_{0}}{\partial y_{2}}=\mathcal{A} \nabla_{y} v_{0}
$$

and thus from equations (61) and (60), it can be seen that

$$
\nabla_{x} \cdot\left(\overline{\overline{\mathbf{A} \mathcal{A} \mathcal{B}^{z^{y}}}} \nabla_{x} p_{0}\right)=\nabla_{x} \cdot\left(\overline{\overline{\boldsymbol{b}-\mathbf{A} \nabla_{z} u_{0}-\mathbf{A} \mathcal{A} \nabla_{y} v_{0}^{z^{y}}}}\right)
$$


By defining

$$
\begin{aligned}
\mathbf{A}_{0}(x) & =\overline{\overline{\mathbf{A} \mathcal{A B}}}^{z} \\
\boldsymbol{b}_{0}(x) & ={\overline{\boldsymbol{b}-\mathbf{A} \nabla_{z} u_{0}-\mathbf{A} \mathcal{A} \nabla_{y} v_{0}}}^{y}
\end{aligned}
$$

and inserting in equation (62), it is finally obtained that

$$
\begin{gathered}
\nabla_{x} \cdot\left(\mathbf{A}_{0}(x) \nabla_{x} p_{0}(x)\right)=\nabla_{x} \cdot \boldsymbol{b}_{0}(x) \text { in } \Omega \\
p_{0}(x)=0 \quad \text { on } \partial \Omega
\end{gathered}
$$

where $\mathcal{A}$ is defined as in equation (45) and $\mathcal{B}$ is defined as in equation (58). In other words, equation (64) is the reiterated homogenized boundary value problem corresponding to the deterministic boundary value problem given by equation (1). 


\section{JET426}

\section{Queries}

A Almqvist, E K Essel, J Fabricius, and P Wall

Q1 IMechE style for matrix is bold roman and for vector is bold italic. Please check whether we have identified all instances correctly.

Q2 References are renumbered in order to maintain the order of citations. Please check whether the references and the citations are correct.

Q3 Figures 7 and 8 are not in their initial order of citations. Please check.

Q4 Reference 'Cioranescu and Donato' has been moved to bibliography section since it is not cited in the text. Please confirm whether this is correct. 